\title{
Kaonic helium atoms in relation to kaonic nuclear bound states
}

\author{
Yoshinori Akaishi ${ }^{a, b}$

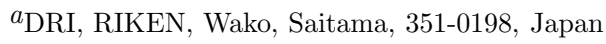

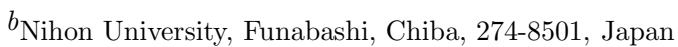

\begin{abstract}
A long-standing problem of $K^{-}{ }^{4} \mathrm{He}$ atom, an abnormal $2 p$ level shift due to strong interaction, is investigated from coupled-channel point of view. An anomalous upward shift of $\Delta E_{2 p}^{\mathrm{cc}}=-11 \mathrm{eV}$ and $\Gamma_{2 p}^{\mathrm{cc}}=21 \mathrm{eV}$ is obtained. The concept of "proximity shift" is introduced, and the effect of centrifugal repulsion in the decay channel is discussed. The atomic $2 p$-level shift measurement will provide a crucial information concerning $p$-wave kaonic nuclear bound state, which reinforces the picture that the $S^{0}(3115)$ and $S^{+}(3140)$ tribaryons are dense $\bar{K}$ nuclei.
\end{abstract}

\section{Introduction}

There has been existing a difficult problem with the $\bar{K}$ optical potential in reproducing the measured $2 p$ level shift of the $K^{-}{ }_{-}^{4} \mathrm{He}$ atom. The data of the three early experiments $[1,2,3]$ are averaged to be

$$
\Delta E_{2 p}^{\exp }=-43 \pm 8 \mathrm{eV}, \quad \Gamma_{2 p}^{\exp }=55 \pm 34 \mathrm{eV},
$$

while theoretical estimates are on the order of

$$
\Delta E_{2 p}^{\mathrm{opt}}=-0.2 \mathrm{eV}, \quad \Gamma_{2 p}^{\mathrm{opt}}=2 \mathrm{eV}
$$

which was given by Batty [4], where the minus sign means an upward shift. The imaginary part of the optical potential is determined from global fits to all the available data of kaonic atoms to be of the order of several tens $\mathrm{MeV}$. As long as this strong imaginary part is employed, one can not get any magnitude of the shift larger than $\sim 2 \mathrm{eV}$ with the optical potential $[4,5]$. Thus, the serious discrepancy remains between experiments and theories. Batty [4] claimed that it is important that the experimental results should be checked and measurements of improved accuracy obtained. 
In this paper a theoretical possibility to reconcile with the anomalous $2 p$ shift will be given based on a coupled channel picture. Here, the anomalous shift is defined by $\left|\Delta E_{2 p}\right|>5 \mathrm{eV}$, where the criterion is taken in enough safety from the value which is impossible to be reproduced by any optical potential with the global-fit imaginary part. On the other hand, our coupled channel model can produce an anomalous shift of the order of $10 \mathrm{eV}$. If the anomalous shift is the actual case, a $2 p$ kaonic nuclear bound state exists. Such a nuclear state has been discussed by Kudrjavtsev [6]. However, a key problem to be solved first must be to overcome the difficulty due to the strong imaginary part of the optical potential. We solve this problem by using coupled channel model, and then discuss about the $p$-wave kaonic nuclear state. The possible existence of the $p$-wave nuclear bound state means that the $\bar{K}$-nucleus potential is strongly attractive enough to accommodate an $s$-wave kaonic nuclear state, which is possibly assigned to the tribaryon $S^{+}(3140)$ reported by Iwasaki et al. [7].

\section{Coupled channel model}

\subsection{Optical model calculation}

As a reference for our coupled-channel model investigation, we study cases of the optical model. The optical potential for $K^{-}{ }^{4} \mathrm{He}$ is taken to be

$$
\begin{array}{r}
U^{\mathrm{opt}}(r)=-\left(U_{0}+i W_{0}\right) F(r), \\
F(r)=\frac{1+w_{0}\left(r / R_{0}\right)^{2}}{1+\exp \left(\left(r-R_{0}\right) / a_{0}\right)}, \\
w_{0}=0.517, R_{0}=0.964 \mathrm{fm} \text { and } a_{0}=0.322 \mathrm{fm},
\end{array}
$$

where $F(r)$ is taken from the density distribution of ${ }^{4} \mathrm{He}$ [8]. The energy of $K^{-}$is obtained by solving a Klein-Gordon equation of

$$
\left\{-(\hbar c)^{2} \vec{\nabla}^{2}+2 \mu c^{2}\left(V_{\mathrm{C}}+U^{\mathrm{opt}}-\epsilon\right)-\left(V_{\mathrm{C}}-\epsilon\right)^{2}\right\} \Psi=0,
$$

where $\mu$ is the reduced mass and $V_{\mathrm{C}}$ is a finite-size Coulomb potential.

The calculated results are shown in Table 1 . The "deep" potential strength $U_{0}^{\text {deep }}$ is searched so as to give a maximum shift of $\left|\Delta E_{2 p}\right|$, and the "shallow" potential strength $U_{0}^{\text {shallow }}$ is taken to be a half of $U_{0}^{\text {deep }}$. We take the case of $W_{0}=60 \mathrm{MeV}$ as a standard, by considering the values of $W_{0}=60 \mathrm{MeV}$ from a modern theory [9] and $W_{0}=70 \mathrm{MeV}$ from a global fit [10]. Thus, the standard case of the deep optical potential is

$$
\Delta E_{2 p}^{\mathrm{opt}}=-1.5 \mathrm{eV}, \quad \Gamma_{2 p}^{\mathrm{opt}}=4 \mathrm{eV} .
$$

The experimental data of Eq.(1) is only reproduced when the imaginary potential is of $2 \sim 3 \mathrm{MeV}$, which is extremely weak compared with the standard, as already stated in Ref.[4].

The deep potential has a depth of $200 \sim 300 \mathrm{MeV}$, which accommodates a $p$-wave nuclear bound state and, as a result, gives an upward shift. The shallow potential has no nuclear bound state and gives definitely a downward shift. When the magnitude of 
Table 1: $2 p$ level shifts of the $K^{-}{ }_{-}^{4}$ He atom calculated for given $W_{0} . U_{0}^{\text {deep }}$ is searched so as to give a maximum shift of $\left|\Delta E_{2 p}\right| . U_{0}^{\text {shallow }}$ is taken to be a half of $U_{0}^{\text {deep }}$.

\begin{tabular}{|c|c|c|c|c|c|c|}
\hline$W_{0}$ & $U_{0}^{\text {deep }}$ & $\Delta E_{2 p}^{\max }$ & $\Gamma$ & $U_{0}^{\text {shallow }}$ & $\Delta E_{2 p}$ & $\Gamma$ \\
\hline 2 & 239 & -43.9 & 70 & 120 & 1.05 & 0.06 \\
\hline 20 & 256 & -4.57 & 9.3 & 128 & 1.13 & 0.69 \\
\hline 40 & 274 & -2.27 & 5.1 & 137 & 1.10 & 1.5 \\
\hline 60 & 289 & -1.47 & 3.8 & 145 & 0.94 & 2.1 \\
\hline 80 & 300 & -1.05 & 3.4 & 150 & 0.67 & 2.5 \\
\hline 100 & 309 & -0.80 & 3.1 & 154 & 0.45 & 2.7 \\
\hline
\end{tabular}

$W_{0}$ and $U_{0}$ in $\mathrm{MeV}, \Delta E_{2 p}$ and $\Gamma$ in $\mathrm{eV}$.

the $2 p$-level shift exceeds $5 \mathrm{eV}$, we propose to call it "anomalous shift" by considering the $W_{0}=20 \mathrm{MeV}$ case in Table 1 to be almost the weakest limit of the imaginary potential:

$$
\text { Anomalous shift; } \quad\left|\Delta E_{2 p}\right| \geq 5 \mathrm{eV} \text {. }
$$

\subsection{Coupled-channel model calculation}

The $K^{-}{ }^{4} \mathrm{He}$ atomic system decays into $\pi+{ }_{Y}^{4} \mathrm{He}(\mathrm{H})$ channels. The coupled channel potential is taken as

$$
U_{\mathrm{D}}(r)=-U_{0} F(r), \quad U_{\mathrm{C}}(r)=U_{\text {coupl }} F(r)
$$

with the same $F(r)$ of Eq.(5), where $\mathrm{D}$ and $\mathrm{C}$ denote diagonal and coupling terms of the potential, respectively.

Table 2: $2 p$ level shifts of the $K^{-}{ }^{4} \mathrm{He}$ atom calculated for given $U_{\text {coupl }} \cdot U_{0}^{\text {deep }}$ is searched

\begin{tabular}{c|ccc|ccc}
\multicolumn{6}{c}{ so as to give a maximum shift of $\left|\Delta E_{2 p}\right| . U_{0}^{\text {shallow }}$ is taken to be a half of $U_{0}^{\text {deep }}$} \\
\hline$U_{\text {coupl }}$ & $U_{0}^{\text {deep }}$ & $\Delta E_{2 p}^{\max }$ & $\Gamma$ & $U_{0}^{\text {shallow }}$ & $\Delta E_{2 p}$ & $\Gamma$ \\
\hline 60 & 233 & -41.9 & 72 & 117 & 1.08 & 0.09 \\
80 & 230 & -24.0 & 43 & 115 & 1.11 & 0.17 \\
100 & 226 & -15.6 & 29 & 113 & 1.16 & 0.27 \\
120 & 221 & -11.0 & 21 & 111 & 1.23 & 0.43 \\
140 & 215 & -8.24 & 16 & 108 & 1.30 & 0.63 \\
160 & 208 & -6.46 & 12 & 104 & 1.38 & 0.90 \\
\hline
\end{tabular}

$U_{\text {coupl }}$ and $U_{0}$ in $\mathrm{MeV}, \Delta E_{2 p}$ and $\Gamma$ in $\mathrm{eV}$. 
The coupled-channel Klein-Gordon equation is written as

$$
\begin{array}{r}
\left\{-(\hbar c)^{2} \vec{\nabla}^{2}+2 \mu c^{2}\left(V_{\mathrm{C}}+U_{\mathrm{D}}-\epsilon\right)-\left(V_{\mathrm{C}}-\epsilon\right)^{2}\right\} \Psi+2 \mu c^{2} U_{\mathrm{C}} \Phi=0, \\
\left\{-(\hbar c)^{2} \vec{\nabla}^{2}+2 \mu^{\prime} c^{2}(Q-\epsilon)-(Q-\epsilon)^{2}\right\} \Phi+2 \mu^{\prime} c^{2} U_{\mathrm{C}} \Psi=0, \\
Q=M_{\Sigma} \mathrm{He}^{2} c^{2}+m_{\pi^{-}} c^{2}-M_{4} \mathrm{He} c^{2}-m_{\mathrm{K}^{-}} c^{2},
\end{array}
$$

where, for simplicity, the decay channel is only limited to the $\pi^{-}+{ }_{\Sigma}^{4} \mathrm{He}[11,12]$ channel of the reduced mass $\mu$ ' with no diagonal potential. For a given $U_{\text {coupl }}$ the diagonal strength $U_{0}$ is searched so as to get a maximum shift of $\left|\Delta E_{2 p}\right|$ in order to obtain the "deep" potential. The "shallow" potential is taken to be a half of the deep potential as before. The obtained results are given in Table 2.

\section{Discussions}

Overall feature of atomic $2 p$-level shift and width is shown in Figure 1 together with a schematic behavior of atomic and nuclear poles on the complex $k$-plane. When the nuclear pole comes closer to the atomic pole, it gives a fluctuation to the atomic level. Here, we introduce a concept of "proximity shift" which is caused when the nuclear pole approaches the $2 p$ atomic pole in the range of its width.

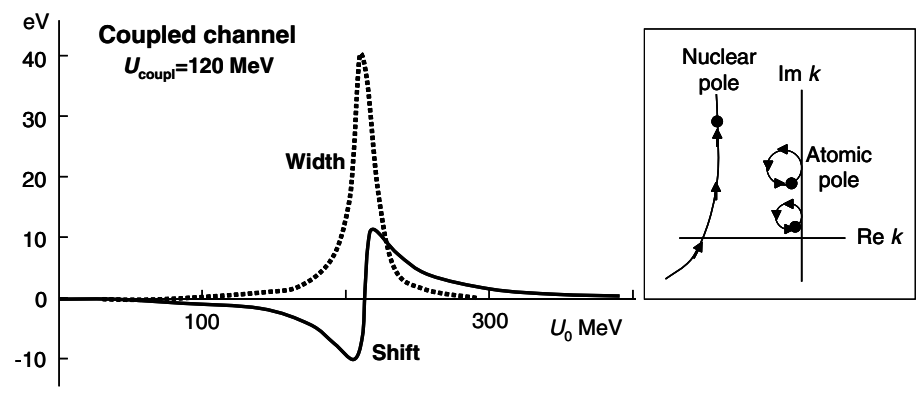

Figure 1: Overall feature of a $2 p$-level complex shift of $K^{-}{ }^{4} \mathrm{He}$ as a function of the potential strength $U_{0}$. Related behaviors of nuclear and atomic poles are shown schematically. The nuclear pole fluctuates the atomic level on passing through.

Figure 2 compares two cases of the $K^{-}{ }_{-}^{4} \mathrm{He}$ atomic $2 p$ level shifts: one is of coupled channel and the other is of optical one. Off the "proximity" region the two cases are very close each other, giving an equivalence between the coupled channel model with $U_{\text {coupl }}=120 \mathrm{MeV}$ and the optical model with $W_{0}=60 \mathrm{MeV}$. In the "proximity" region, however, $2 p$ level shifts from the two models are extremely different: the maximum shift of the coupled channel model is almost 10 times larger than that of the optical one. The standard of the coupled-channel deep potential is set to

$$
\Delta E_{2 p}^{\mathrm{cc}}=-11 \mathrm{eV}, \quad \Gamma_{2 p}^{\mathrm{cc}}=21 \mathrm{eV} .
$$




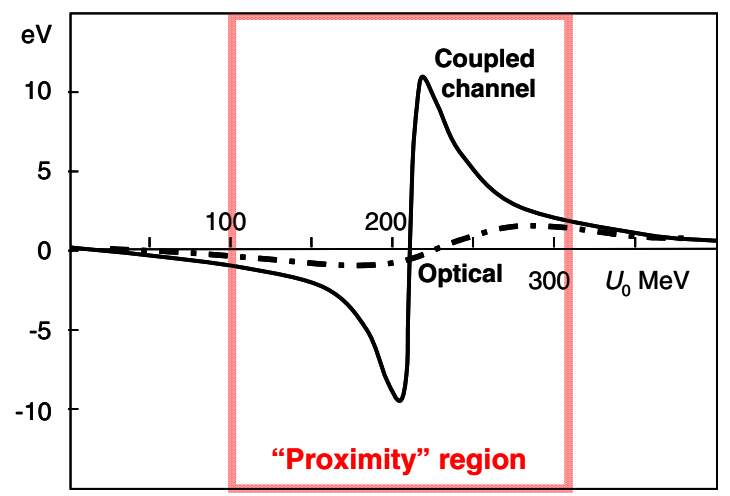

Figure 2: $2 p$ level shifts of the $K^{-}{ }^{4} \mathrm{He}$ atom calculated with coupled-channel and optical models. The solid line and the dot-dashed line are for coupled-channel one with $U_{\text {coupl }}=120 \mathrm{MeV}$ and for optical one with $W_{0}=60 \mathrm{MeV}$, respectively. The "proximity" region is denoted. The maximum shift of "coupled channel" is almost one-order of magnitude larger than that of "optical".

The deep potential is of $200 \sim 250 \mathrm{MeV}$ strength, and has a $p$-wave nuclear bound state, bringing an upward shift. The shallow potential has no such bound state and gives definitely a downward shift. It is shown that a set of deep coupled-channel potentials having a $p$-wave $\bar{K}$ nuclear bound state can cause an anomalously large magnitude of $2 p$ level shift.

What is the origin of the large difference between the coupled-channel model and the optical model? The centrifugal potential in the $\pi+{ }_{\Sigma}^{4} \mathrm{He}$ decay channel is essentially relevant to the difference. Since the pion is of small mass, a large centrifugal repulsion appears and it suppresses the $p$-wave decay into this channel. Table 3 shows the centrifugal effect. The third row is the case of no centrifugal potential. The maximum shift, the width and the position of $p$-wave nuclear pole are surprisingly close to those of the optical potential which are denoted in the fourth row. When the centrifugal potential is switched on, the maximum $2 p$-level shift amounts to an anomalous value, $-11 \mathrm{eV}$, due to a close proximity of the $p$-wave nuclear pole to the $2 p$ atomic pole as seen in the last column. If the early data of Eq.(1) is correct, we need much stronger repulsion as indicated in the first row, a part of which could come from $\pi$-nucleus strong interaction. The nuclear pole position resembles that of Ref.[6] in this case.

This decay-channel centrifugal effect clearly shows a limitation of so-called "global-fit" approach [10] done by using all the available kaonic atom data on a same footing. Global optical potentials with $l$-independent imaginary part cannot reproduce any anomalous $l \neq 0$ level shift which appears in the "proximity" region. The global-fit optical potential should not be applied at least to the problem of the $2 p$ level shifts of the $K^{-}{ }^{3,4} \mathrm{He}$ atoms. 
Table 3: Effect of the centrifugal repulsion in the $\pi+{ }_{\Sigma}^{4}$ He decay channel. The $2 p$ level shift of the $K^{-}{ }^{4} \mathrm{He}$ atom is largely affected by the centrifugal potential, the strength of which is controlled with a factor $f_{\mathrm{cf}}$ as $f_{\mathrm{cf}} V_{\text {centrifugal }}$.

\begin{tabular}{ccc|ccc}
\hline$U_{\text {coupl }}$ & $f_{\text {cf }}$ & $U_{0}^{\text {deep }}$ & $\Delta E_{2 p}^{\max }$ & $\Gamma$ & $(E, \Gamma)_{p-\text { pole }}^{\text {nucl }}$ \\
\hline 120 & 2 & 224 & -46 & 91 & $(-0.3,0.7)$ \\
120 & 1 & 221 & -11 & 21 & $(-1.5,3.8)$ \\
120 & 0 & 259 & -1.4 & 3.0 & $(-13,35)$ \\
\hline$W_{0}=60$ & - & 289 & -1.5 & 3.8 & $(-12,44)$ \\
\hline \multicolumn{7}{c}{$U_{\text {coupl }}\left(W_{0}\right)$ and $(E, \Gamma)^{\text {nucl }}$ in $\mathrm{MeV}, \Delta E_{2 p}$ and $\Gamma$ in $\mathrm{eV}}$.
\end{tabular}

\section{1 $\bar{K}$ nuclear bound state}

It is known that the $\bar{K} N$ interaction is strongly attractive in the $T=0$ state below the $\Lambda(1405)$ resonance and brings about a dropping $K^{-}$mass in nuclear matter $[13,14]$. This is connected to an exciting issue of whether kaon condensation in dense nuclear matter like compact star could occur $[15,16,17]$. Recently, few-body $\bar{K}$ nuclear systems have been investigated theoretically $[18,19,20,21]$ (see also [22] as an early work) based on phenomenological $\bar{K} N$ interaction which reproduces low-energy scattering data [23], kaonic hydrogen data $[24,25]$ (see also [26] as the most recent data) and the binding energy and width of $\Lambda(1405)$.

Figure 3 shows the potentials of $K^{-}{ }^{3} \mathrm{He}$ and $K^{-}{ }^{4} \mathrm{He}$ calculated in Ref.[18]. Since a deeply bound $\bar{K}$ contracts the nucleon core, the kaonic nuclei give us potential information about the shrunk-core cases denoted by the solid lines. On the other hand, the kaonic atoms provide information on the unchanged-core potentials shown by the dotted lines, which are more directly related to the free $\bar{K} N$ interaction with less medium modifications. The potential depths come around $\sim 270 \mathrm{MeV}$ as pointed by the arrows. Figure 4 shows the calculated $2 p$ level shifts of the $K^{-}{ }^{4} \mathrm{He}$ and $K^{-}{ }^{3} \mathrm{He}$ atoms. $U_{0}=200 \sim 300 \mathrm{MeV}$ region just corresponds to the "proximity" region. We could expect an anomalous $2 p$ upward shift for $K^{-}{ }^{4} \mathrm{He}$ and an anomalous $2 p$ downward shift for $K^{-}{ }^{3} \mathrm{He}$. Experimental observation of such anomalous shifts would pin down the strength of very attractive $\bar{K}$ nucleus potentials.

Recently, an experimental indication was reported on the predicted $T=0 \mathrm{ppnK} K^{-}[18]$ from ${ }^{4} \mathrm{He}$ (stopped $K^{-}, n$ ) experiment at KEK by Iwasaki et al. [7]. The observed bound state, $S^{+}(3140)$ tribaryon, lies much deeper than the theoretical prediction. More recently, Suzuki et al. [27] has discovered pnnK ${ }^{-}$, named $S^{0}(3115)$ tribaryon, from ${ }^{4} \mathrm{He}$ (stopped $\left.K^{-}, p\right)$ experiment. The binding energies of the two tribaryons are accounted for within the framework of the predicted $\bar{K}$ clusters by Akaishi, Dote and Yamazaki [28], where the relativistic effect and an enhanced bare $K N$ interaction by $15 \%$ in the dense nuclear medium are taken into account. 

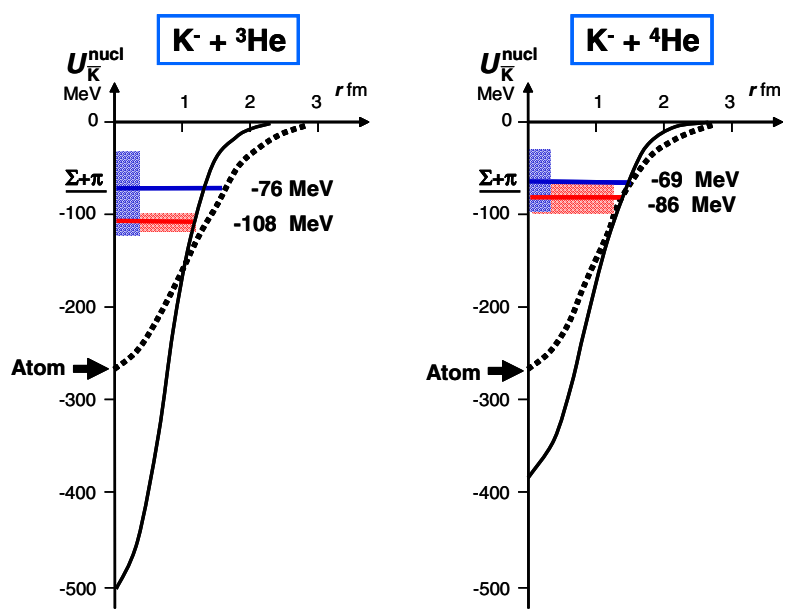

Figure 3: Real-part potentials of $K^{-}{ }^{3} \mathrm{He}$ and $K^{-}{ }^{4} \mathrm{He}$ strong interaction. The solid and dotted lines are the cases of shrunk and unchanged nucleon cores, respectively. Nuclear $s$-wave bound states are shown. Atomic level shifts provide information about the potential strength from the unchanged cores as indicated by the arrows.

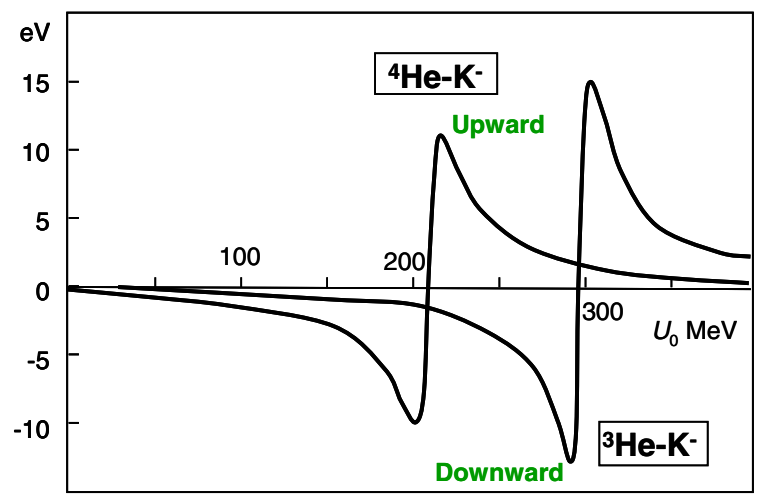

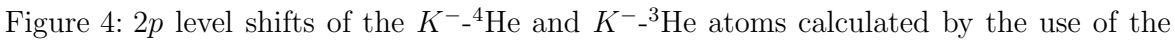
coupled-channel model with $U_{\text {coupl }}=120 \mathrm{MeV}$. 


\section{Conclusions}

A coupled channel model is applied to the $2 p$ level shift problem of the $K^{-}{ }_{-}^{4} \mathrm{He}$ and $K^{-}$${ }^{3} \mathrm{He}$ atoms. A reasonable prediction done in this paper could be an anomalous upward shift of $\Delta E_{2 p}^{\mathrm{cc}}=-11 \mathrm{eV}$ and $\Gamma_{2 p}^{\mathrm{cc}}=21 \mathrm{eV}$ for the $K^{-}{ }_{-}^{4} \mathrm{He}$ atom, and also an anomalous downward shift for the $K^{-}{ }^{3} \mathrm{He}$ atom. The optical potential approach with "global fit" breaks down in the anomalous "proximity shift" case.

If such anomalous $2 p$-level shifts of $K^{-}$-He atoms are established experimentally, valuable information concerning deeply-bound kaonic nuclear systems would be obtained: a strongly attractive potential which accommodates nuclear $\bar{K}$ states is unambiguously justified. It reinforces the picture that the tribaryons, $S^{0}(3115)$ and $S^{+}(3140)$, are dense $\bar{K}$ nuclear states, which would develop to important issues of chiral symmetry restoration $[29,30,31]$, kaon condensation, possible existence of strange matter and so on.

\section{References}

[1] C.E. Wiegand and R.H. Pehl, Phys. Rev. Lett. 27 (1971) 1410.

[2] C.J. Batty, S.F. Biagi et al., Nucl. Phys. A 326 (1979) 455.

[3] S. Baird et al., Nucl. Phys. A 392 (1983) 297.

[4] C.J. Batty, Nucl. Phys. A 508 (1990) 89c.

[5] R. Seki, Phys. Rev. C 5 (1972) 1196.

[6] A.E. Kudrjavtsev, V.D. Mur and V.S. Popov, Phys. Lett. 143B (1984) 41.

[7] M. Iwasaki et al., arXiv:nucl-ex/0310018.

[8] Nuclear Data Tables 14 (1974) 485.

[9] A. Cieply, E. Friedman, A. Gal and J. Mares, Nucl. Phys. A 696 (2001) 173.

[10] C.J. Batty, E. Friedman and A. Gal, Phys. Rep. 287 (1997) 385.

[11] T. Harada, S. Shinmura, Y. Akaishi and H. Tanaka, Nucl. Phys. A 507 (1990) 715.

[12] T. Nagae et al., Phys. Rev. Lett. 80 (1998) 1605.

[13] T. Waas, N. Kaiser and W. Weise, Phys. Lett. B 365 (1996) 12; B 379 (1996) 34.

[14] W. Weise, Nucl. Phys. A 610 (1996) 35c.

[15] D.B. Kaplan and A.E. Nelson, Phys. Lett. B 175 (1986) 57.

[16] G.E. Brown, Nucl. Phys. A 574 (1994) 217c.

[17] G.E. Brown and H.A. Bethe, Astrophys. J. 423 (1994) 659.

[18] Y. Akaishi and T. Yamazaki, Phys. Rev. C 65 (2002) 044005.

[19] T. Yamazaki and Y. Akaishi, Phys. Lett. B 535 (2002) 70.

[20] A. Dote, H. Horiuchi, Y. Akaishi and T. Yamazaki, Phys. Lett. B 590 (2004) 51.

[21] T. Yamazaki, A. Dote and Y. Akaishi, Phys. Lett. B 587 (2004) 167.

[22] S. Wycech, Nucl. Phys. A 450 (1986) 399c.

[23] A.D. Martin, Nucl. Phys. B 179 (1981) 33.

[24] M. Iwasaki et al., Phys. Rev. Lett. 78 (1997) 3067.

[25] T.M. Itoh et al., Phys. Rev. C 58 (1998) 2366.

[26] C. Guaraldo et al., Eur. Phys. J. A 19 (2004) 185.

[27] T. Suzuki et al., Phys. Lett. B 597 (2004) 263. 
[28] Y. Akaishi, A. Dote and T. Yamazaki, Phys. Lett. B 613 (2005) 140.

[29] T. Hatsuda and T. Kunihiro, Phys. Rev. Lett. 55 (1985) 158.

[30] W. Weise, Nucl. Phys. A 553 (1993) 59c.

[31] P. Kienle and T. Yamazaki, Prog. Part. Nucl. Phys. 52 (2004) 85. 
